\title{
Mycobacterium abscessus infection in a spa worker
}

\author{
Miguel Costa-Silva ${ }^{1 凶}$, Artur César ${ }^{1}$, Nuno Preto Gomes ${ }^{1}$, Filomena Azevedo ${ }^{1}$
}

\begin{abstract}
Mycobacterium abscessus (M. abscessus) is a non-tuberculous mycobacterium widely present in the natural environment and is now being increasingly identified as a cause of human disease. However, it can cause skin and soft tissue infection following trauma or surgery. We report a case of a skin ulcer associated with $M$. abscessus in a spa worker. Frequently, contamination with atypical mycobacteria occurs through tap water. However, in most outbreaks the infection source remains unknown. For an early diagnosis, it is important to perform biopsies and mycobacterium cultures. No specific treatment for skin infection with $M$. abscessus has been established; it depends on the immune status of the host and the extent of disease. Although it is rare, non-tuberculous mycobacterial infection should be part of the differential diagnosis of cutaneous ulcers, especially on trauma-prone areas.
\end{abstract}

Keywords: Mycobacterium abscessus, ulcer, spa worker

Received: 30 January 2018 | Returned for modification: 16 March 2018 | Accepted: 30 March 2018

\section{Introduction}

Mycobacterium abscessus (M. abscessus) is a rapidly growing mycobacterium widely present in the natural environment, such as in the soil and water $(1,2)$. It is increasingly being identified as a cause of human disease, particularly as an agent of cutaneous and soft-tissue infections, pulmonary infections, and disseminated disease associated with immunosuppression $(3,4)$. In recent decades, increasing incidence of non-tuberculous (also called "atypical") mycobacteria infections has been reported, and those caused by M. abscessus are no exception (4). The increasing frequency of immunosuppression and surgical procedures, and the more efficient identification of the pathogen could explain this increase $(1,5)$.

\section{Case description}

A 24-year-old woman was referred to our clinic with a 3-month history of a skin ulcer. The patient, a spa worker, did not recall any skin injury and had an unremarkable medical history. She reported a total weight loss of $8 \mathrm{~kg}$ over the previous 2 months and anorexia, but reported no asthenia, fever, or night sweats. Clinical examination identified a $4 \mathrm{~cm}$ wide ulcer with small central pustules and raised border on the anterolateral aspect of the right thigh. In addition, a mobile and painful subcutaneous nodule with overlying erythema was identified in the ipsilateral inguinal region (Fig. 1). Laboratory tests were normal/negative, including human immunodeficiency virus and hepatitis antibodies, as well as a venereal disease research laboratory test. The histopathological examination of a biopsy specimen from the cutaneous ulcer revealed an abundant polymorphic inflammatory infiltrate with epithelioid granuloma formation. Acid-fast, PAS, and Gram stains were all negative. Cultural examination of an aspirate specimen from the inguinal adenopathy identified M. abscessus, but we were not able to determine $M$. abscessus subspecies because at that time subspecies identification was not available in our hospital. Antimicrobial susceptibility tests concluded that it was resistant to trimethoprim/sulfamethoxazole and $\beta$-lactam antibiotics, and the patient was treated with clarithromycin 1,00o mg/day, levofloxacin $750 \mathrm{mg} /$ day, and amikacin 1,000 mg/day for 3 months followed by clarithromycin and levofloxacin for another 3 months with complete resolution of the lesions (Fig. 2). The patient is free of disease 6 months after stopping treatment. Local public health authorities were contacted and, after collecting multiple samples including water samples, they were able to eradicate the focus of contamination.

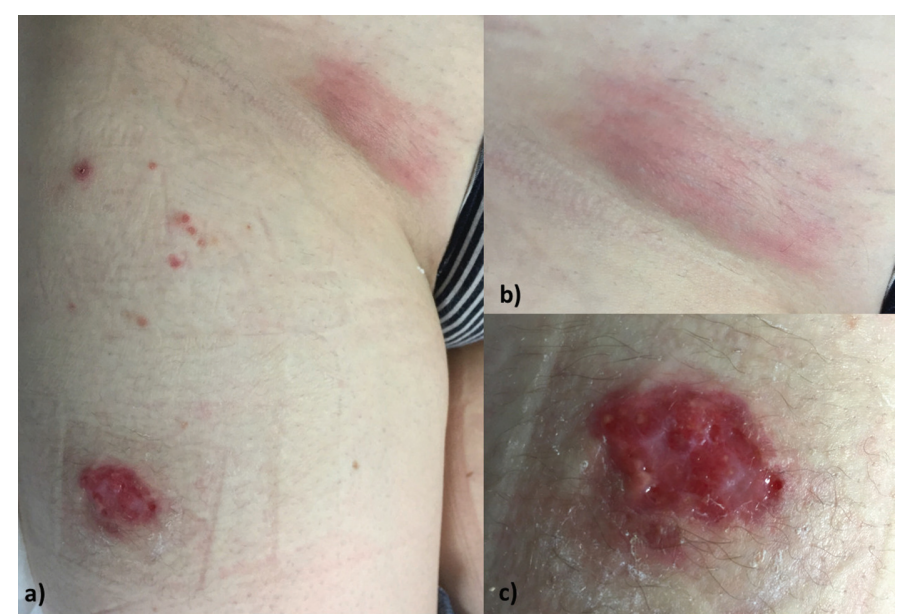

Figure 1 | Ulcer of the right thigh $(\mathrm{a}, \mathrm{c})$ and inguinal adenopathy $(\mathrm{a}, \mathrm{b})$.

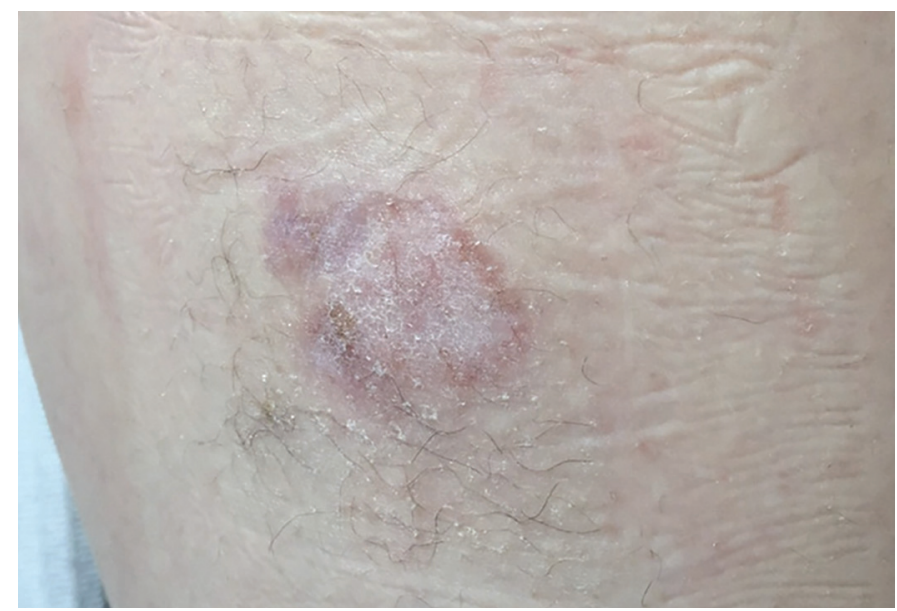

Figure 2 | Complete resolution after treatment. 


\section{Discussion}

The nomenclature of rapidly growing mycobacteria has recently been changed. Initially, they were included in the $M$. fortuitum complex but, with the advent of biochemical techniques, four separate species associated with different clinical conditions and a distinct profile of antimicrobial susceptibility have been identified: M. peregrinum, M. chelonae, M. abscessus, and M. fortuitum (3). Recently the $M$. abscessus complex was divided into three subspecies: M. abscessus subsp. abscessus, M. abscessus subsp. massiliense, and M. abscessus subsp. Bolletii.

Cutaneous infections with $M$. abscessus usually occur by inoculation of the bacteria following trauma of the skin with contaminated devices such as inadequately sterilized medical equipment and after mesotherapy, tattooing, acupuncture, and insulin injections $(1,2)$. Frequently, contamination with non-tuberculous mycobacterium occurs through tap water $(5,7)$. However, in most outbreaks, as well as in our case, the infection source remains unknown.

For an early diagnosis, it is important to perform biopsies and mycobacterium cultures $(4,8)$. The immune status of the host is the single most important factor determining the course of the disease $(3,9)$. No specific treatment for skin infection with $M$. abscessus has been established because it depends on the immune status of the host and the extent of disease $(3,9)$. Multiple treatment modalities are recommended, including surgery and a long course of antibiotics that can be used in combination (1). $M$. abscessus is inherently resistant to current first-line antituberculosis drugs, but recently rifabutin has shown potential efficacy $(7,10)$. Due to both natural and acquired resistance to most currently available antibiotics, combination therapy, which includes macrolide, aminoglycoside, carbapenem, and new quinolones, is frequently used (4). The duration of treatment is not clear but, as with other mycobacterial infections, most authors agree that at least 6 months of treatment should be provided (3).

Rapidly growing atypical mycobacteria post unique challenges to clinicians. Although it is rare, non-tuberculous mycobacterial infection should be part of the differential diagnosis of cutaneous ulcers, especially on trauma-prone areas, as illustrated in this case report.

\section{References}

1. Sousa PP, Cruz RC, Schettini AP, Westphal DC. Mycobacterium abscessus skin infection after tattooing-case report. An Bras Dermatol. 2015;90:741-3.

2. Bates TR, Keenher T, O'Reilly LC, Heath CH, Flexman JP, Murray RJ. Extensive cutaneous Mycobacterium abscessus infection due to contaminated insulin delivery system. QJM. 2009;102:881-4.

3. Kwon YH, Lee GY, Kim WS, Kim KJ. A case of skin and soft tissue infection caused by Mycobacterium abscessus. Ann Dermatol. 2009;21:84-7.

4. Kishida D, Sato M, Kobayashi C, Ueno KI, Kinoshita T, Kodaira M, et al. Intractable cutaneous nontuberculous mycobacteriosis (Mycobacterium abscessus) during treatment for systemic lupus erythematosus. Intern Med. 2017;56:1253-7.

5. Nessar R, Cambau E, Reyrat JM, Murray A, Gicquel B. Mycobacterium abscessus: a new antibiotic nightmare. J Antimicrob Chemother. 2012;67:810-8.

6. Ryu HJ, Kim WJ, Oh CH, Song HJ. latrogenic Mycobacterium abscessus infection associated with acupuncture: clinical manifestations and its treatment. Int J Dermatol 2005;44:846-50.
7. Sabin AP, Ferrieri P, Kline S. Mycobacterium abscessus complex infections in children: a review. Curr Infect Dis Rep. 2017;19:46.

8. Rodriguez G, Ortegon M, Camargo D, Orozco LC. Iatrogenic Mycobacterium abscessus infection: histopathology of 71 patients. Br J Dermatol. 1997;137:214-8.

9. Conaglen PD, Laurenson IF, Sergeant A, Thorn SN, Rayner A, Stevenson J. Systematic review of tattoo-associated skin infection with rapidly growing mycobacteria and public health investigation of a cluster in Scotland, 2010. Euro Surveill. 2013;18:20553.

10. Aziz DB, Low JL, Wu ML, Gengenbacher M, Teo JWP, Dartois V, et al. Rifabutin is active against Mycobacterium abscessus complex. Antimicrob Agents Chemother. 2017;61. [Epub ahead of print]. 